Introduction EXCELLENT (NCT03685578) is a prospective, single-arm, multicenter, real-world international registry of mechanical thrombectomy (MT) for stroke with the EmboTrap device as first line treatment. The study entails thrombus analysis of specimens collected with each MT pass.

Aim of the Study To compare rates of mRS 0-2 at 90 days and clot characteristics in subjects with and without first pass effect (FPE).

Methods FPE was defined as mTICI $2 \mathrm{c} / 3$ after one pass and non-FPE as mTICI $2 \mathrm{c} / 3$ after $>1$ pass as adjudicated by an independent core lab. Clot analysis was performed by independent central labs blinded to clinical data. mRS at 90 days was scored by investigators blinded to procedural data.

Results Overall mTICI2c/3 rates were 63.7\% (326/512). FPE was achieved in $37.1 \%(190 / 512)$ and non-FPE in $26.6 \%$ (136/512) subjects. 90 day $\mathrm{mRS} 0-2$ or equal to pre-stroke was achieved in 47.2\% (75/159) with FPE and in 42.1\% (51/ 121) non-FPE patients. All-cause 90-day mortality was $19.1 \%$ (34/178) in subjects with FPE and 26.4\% (34/129) in subjects with non-FPE. Major thrombus components (mean\% \pm SD) were as follows: RBC: FPE 45.88 \pm 20.54 , non-FPE 39.08 \pm 18.23 , and first pass $\mathrm{mTICI}<2 \mathrm{c} / 3$ 40.96 \pm 18.84 ; Fibrin: FPE $24.72 \pm 13.82$, non-FPE $29.09 \pm 15.76$ first pass $\mathrm{mTICI}<2 \mathrm{c} / 3$ $29.20 \pm 14.58$

Conclusions The high rate of 'real-world' FPE observed in EXCELLENT was associated with improved clinical outcomes. Clots retrieved with FPE had higher RBC and lower fibrin content compared to non FPE and to first pass mTICI $<2 \mathrm{c} / 3$ e. These preliminary findings await confirmation from analysis of the full dataset.

Disclosure EXCELLENT is sponsored by Cerenovus. Dr. Jovin is a consultant for Neuravi, Codman Neurovascular, Stryker (PI DAWN; unpaid), Fundacio Ictus (PI REVASCAT; unpaid), and holds stock in Anaconda, Silk Road, and Blockade Medical.

\section{EP47* INVESTIGATION OF CURRENT AND NOVEL TREATMENT STRATEGIES FOR ICA AND MCA OCCLUSIONS IN AN IN- VITRO THROMBECTOMY MODEL}

${ }^{1}$ RG Nogueira, ${ }^{2}$ J Thornton, ${ }^{3}$ L Mullins, ${ }^{4} S$ Fitzgerald. ${ }^{1}$ Neurology, Grady Memorial Hospital and Emory University, Atlanta, GA, USA; ${ }^{2}$ Radiology, Beaumont Hospital and Royal College of Surgeons Ireland, Dublin; ${ }^{3}$ Perfuze Ltd; ${ }^{4}$ Physiology, National University of Ireland Galway, Galway, Ireland

\subsection{6/neurintsurg-2021-ESMINT.46}

Introduction Various mechanical thrombectomy devices and techniques including Stentrievers and Aspiration Catheters are used in the treatment of acute ischemic stroke. ${ }^{1}$ Balloon Guide Catheters (BGC) are commonly used to achieve flow control. A new generation of 'Super-bore' 088" catheters are currently being tested clinically. ${ }^{2} 3$

Objective/Aim We evaluated the in-vitro revascularization performance of current and novel thrombectomy approaches.

Methods National University of Ireland Galway Research Ethics Committee approved the study. Clot analogues from human blood were used to create ICA $(20 \mathrm{~mm})$ and MCA $(10 \mathrm{~mm})$ occlusions in an In-vitro thrombectomy model. Thrombectomy setups tested were; ADAPT: Millipede 088, SOFIA, SOFIA+BGC and Combination; Millipede $088+$ Soliitaire, SOFIA+BGC+Solitaire, SOFIA+Solitaire. 10 replicates of each test were performed. Endpoints were First Pass Effect
(FPE), Second and Third-pass success and procedural-related distal emboli from 50-1000 $\mu \mathrm{m}$.

Results The Millipede 088 catheter achieved the highest rate of FPE in both ICA (60\%) and MCA (100\%) occlusions and had the lowest number of procedural-related emboli compared to all other device setups, followed by the Millipede 088 +Solitaire technique. Of the currently used techniques, the use of a Solitaire in combination with a SOFIA lead to a higher rate of FPE in ICA occlusions (40\% vs 20\%, SOFIA $+\mathrm{BGC}+$ Solitaire vs SOFIA + BGC), but a lower rate of FPE in MCA occlusions $(50 \%$ vs $60 \%$, SOFIA+BGC+Solitaire vs SOFIA+BGC).

Conclusions The novel 0.088 " aspiration catheter achieves the best FPE rates in both ICA and MCA occlusions. In terms of existing techniques, ADAPT is more effective in the MCA, while the stentrievers provide a benefit in the ICA.

\section{REFERENCES}

1. Hafeez MU, Kan P, Srivatsan A, Moore S, Jafari M, DeLaGarza C, et al. Comparison of first-pass efficacy among four mechanical thrombectomy techniques: a single-center experience. World Neurosurgery 2020;144:e533-e40.

2. Gershon BS, Bageac DV, Shigematsu T, Majidi S, De Leacy R. Republished: first clinical report of aspiration through a novel 0.088 -inch catheter positioned in the M1 middle cerebral artery for ELVO thrombectomy. Journal of Neurolnterventional Surgery 2021;13(4):e4-e.

3. Nogueira RG, Mohammaden MH, Al-Bayati AR, Frankel MR, Haussen DC. Preliminary experience with 088 large bore intracranial catheters during stroke thrombectomy. Interventional Neuroradiology 2020:1591019920982219.

Disclosure Seán Fitzgerald received research funding from Enterprise Ireland that is co-funded by Perfuze Ltd. Liam Mullins declares the following competing interest; Perfuze (stock options). John Thornton declares the following competing interests; Perfuze (Physician Advisory Board, stock options); Consultancy fees: Microvention, Johnson and Johnsons. Raul G. Nogueira declares the following competing interests: consulting fees for advisory roles with Anaconda, Biogen, Cerenovus, Genentech, Imperative Care, Medtronic, Phenox, Prolong Pharmaceuticals, Stryker Neurovascular and stock options for advisory roles with Astrocyte, Brainomix, Cerebrotech, Ceretrieve, Corindus Vascular Robotics, Vesalio, Viz-AI, and Perfuze.

\section{EP48* SAFETY AND EFFECTIVENESS OF MECHANICAL THROMBECTOMY FOR PRIMARY ISOLATED DISTAL VESSEL OCCLUSIONS: A MONOCENTRIC RETROSPECTIVE COMPARATIVE STUDY}

\begin{abstract}
$1,{ }^{2} \mathrm{M}$ Elhorany, ${ }^{3} \mathrm{C}$ Rosso, ${ }^{4} \mathrm{E}$ Shotar, ${ }^{3} \mathrm{~F}$ Baronnet-Chauvet, ${ }^{4} \mathrm{~K}$ Premat, ${ }^{4} \mathrm{~S}$ Lenck, ${ }^{3} \mathrm{~S}$ Crozier, ${ }^{4} \mathrm{C}$ Corcy, ${ }^{5} \mathrm{~L}$ Bottin, ${ }^{6} \mathrm{O}$ Yassin Mansour, ${ }^{7} \mathrm{E}-\mathrm{S}$ Ali Tag El-din, ${ }^{7} \mathrm{~W}$ Ahmed Fadel, ${ }^{4} \mathrm{~N}$ Antoine Sourour, ${ }^{5} \mathrm{~S}$ Alamowitch, ${ }^{3} Y$ Samson, ${ }^{4} \mathrm{~F}$ Clarençon. ${ }^{1}$ Department of Neuroradiology. PitiéSalpêtrière Hospital, Paris, France; ${ }^{2}$ Department of Neurology. Faculty of Medicine, Tanta University, Tanta, Egypt; ${ }^{3} A P-H P$, Urgences Cérébro-Vasculaires, Hôpital Pitié-Salpêtrière, DMU Neuroscience, 75013; ${ }^{4}$ Department of Neuroradiology, Pitié-Salpêtrière Hospital; ${ }^{5}$ Department of Vascular Neurology, Saint-Antoine Hospital, Paris, France; ${ }^{6}$ Department of Neurology, Faculty of Medicine, Alexandria University, Alexandria; 7 Department of Neurology, Faculty of Medicine, Tanta University, Tanta, Egypt
\end{abstract}

\subsection{6/neurintsurg-2021-ESMINT.47}

Background Distal vessel occlusions represent about 25-40\% of acute ischemic stroke (AIS), either as primary occlusion or secondary occlusion complicating mechanical thrombectomy (MT) for large vessel occlusion.

Objective Our aim was to evaluate safety and effectiveness of MT associated with the best medical treatment (BMT) in the 
management of AIS patients with distal vessel occlusion in comparison with the BMT alone.

Methods Retrospective analysis was conducted on AIS patients treated by MT+BMT for primary distal vessel occlusion between 2015 and 2020, and were compared with a historic cohort managed by BMT alone between 2006 and 2015 selected based on the same inclusion criteria. A secondary analysis was conducted using propensity score matching (PSM) including the following: NIHSS, age and treatment with intravenous thrombolysis (IVT) as covariates.

Results Of 650 patients screened, 44 patients with primary distal vessel occlusions treated by MT+BMT were selected and compared with 36 patients who received BMT alone. After PSM, 28 patients in each group were matched without significant difference. Good clinical outcome defined as mRS $\leq 2$ was achieved by $53.6 \%$ of the MT+BMT group and $57 \%$ of the BMT group (OR, 0.87; 95\%CI, 0.3-2.4; $\mathrm{P}=1.00)$. The mortality rate was comparable in both groups $(7 \%$ vs $10.7 \%$ in $\mathrm{MT}+\mathrm{BMT}$ and BMT patients, respectively; $\mathrm{OR}=0.64 ; 95 \%$ CI, 0.1-4; $\mathrm{P}=1.00)$. Symptomatic intracranial haemorrhage $(\mathrm{ICH})$ was seen in only one patient treated by MT+BMT (3.6\%).

Conclusion Mechanical thrombectomy seems to be comparable with the best medical treatment regarding the effectiveness and safety in the management of patients with distal vessel occlusions.

\section{REFERENCES}

1. Sarraj A, Sangha N, Hussain MS, et al. Endovascular therapy for acute ischemic stroke with occlusion of the middle cerebral artery M2 segment. JAMA Neurol 2016;73:1291-1296.

2. Saver JL, Chapot R, Agid R, et al. Thrombectomy for distal, medium vessel occlusions: a consensus statement on present knowledge and promising directions. Stroke 2020:51:2872-2884

3. Ospel JM, Menon BK, Demchuk AM, et al. Clinical course of acute ischemic stroke due to medium vessel occlusion with and without intravenous alteplase treatment. Stroke 2020;51:3232-3240.

Disclosure Pr F. Clarençon reports conflict of interest with Medtronic, Guerbet, Balt Extrusion, Penumbra (payment for readings), Codman Neurovascular and Microvention (core lab). Dr N. Sourour is consultant for Medtronic, Balt Extrusion, Microvention, Stock/Stock Options: Medina. The other authors report no conflict of interest concerning the materials or methods used in this study or the findings specified in this paper.

\section{EP49* HEMORRHAGIC TRANSFORMATION AND FIRST PASS EFFECT}

${ }^{1} \mathrm{G}$ Meeks, ${ }^{2} \mathrm{O}$ Zaidat, ${ }^{3} \mathrm{~A}$ Hassan, ${ }^{4} \mathrm{~F}$ Fifi, ${ }^{5} \mathrm{~A}$ Yoo, ${ }^{1} \mathrm{~S}$ Sheth. ${ }^{1}$ UTHealth McGovern Medical School, Houston, $T X_{;}{ }^{2}$ Mercy Health St. Vincent Medical Center, Toledo, OH; ${ }^{3}$ University of Texas Rio Grande Valley - Valley Baptist Medical Center, Harlingen, $T X_{;}{ }^{4}$ Icahn School of Medicine at Mount Sinai, New York, NY; ${ }^{5}$ Texas Stroke Institute, Dallas-Fort Worth, TX, USA

\subsection{6/neurintsurg-2021-ESMINT.48}

Achieving substantial reperfusion in the first pass is a strong predictor of clinical outcomes. Its role in preventing symptomatic $\mathrm{ICH}(\mathrm{sICH})$ is incompletely characterized.

We assess the association of increasing number of passes with sICH using a large multicenter, prospectively collected international registry with core-lab adjudicated blinded imaging endpoints.
We analyzed all patients receiving EVT for LVO in the COMPLETE (Penumbra, Inc) registry. SICH at 24 hours was defined as greater than four-point increase in NIHSS associated with $\mathrm{ICH}$ after review by two independent physicians. Multivariable analysis adjusted for age, NIHSS, occlusion location, and ASPECTS were used to assess the likelihood of developing sICH.

Among 650 patients included in the analysis, median age was 70 [IQR 60-79] and 54\% were female. Average number of passes was 1.5. First pass mTICI $2 b-3$ was achieved in $55.5 \%(358 / 645)$ while $32.2 \%(208 / 645)$ required two or greater attempts. SICH occurred in $25(3.8 \%)$ and $\mathrm{PH}-2$ in $20(3.1 \%)$. We identified an increased likelihood of sICH with increasing number of attempts to achieve TICI $2 \mathrm{~b}$ or greater ( 3 vs 1 pass, OR $=3.98$ [95\% CI, 1.05-15.0], and 4 vs 1 pass, OR $=5.04[95 \% \mathrm{CI}, 1.35-18.8])$. Failure to achieve mTICI $2 b$ or greater $(79 / 645)$ was associated with increased incidence of sICH compared to first pass reperfusion $(\mathrm{OR}=$ 4.66, [CI 95\%, 1.43-15.1]).

Achieving substantial reperfusion with the fewest number of thrombectomy attempts was associated with decreasing likelihood of sICH.

\section{REFERENCES}

1. Zaidat 00, Castonguay $A C$, Linfante I, et al. First pass effect: a new measure for stroke thrombectomy devices. Stroke 2018;49(3):660-666. doi:10.1161/ STROKEAHA.117.020315

2. Maros ME, Brekenfeld C, Broocks $G$, et al. Number of retrieval attempts rather than procedure time is associated with risk of symptomatic intracranial hemorrhage. Stroke 2021;52(5):1580-1588. doi:10.1161/STROKEAHA.120.031242

Disclosure All COMPLETE trial investigators received support from Penumbra Inc

\section{EP50* CATHETER TIP DISTENSIBILITY SUBSTANTIALLY INFLUENCES THE ASPIRATION FORCE OF THROMBECTOMY DEVICES}

${ }^{1,2}$ J Li, ${ }^{3,4} \mathrm{O}$ Castaño, ${ }^{5} \mathrm{~A}$ Tomasello, ${ }^{5} \mathrm{M}$ De Dios, ${ }^{1,2} \mathrm{P}$ Canals, ${ }^{6,7} \mathrm{E}$ Engel, ${ }^{1,2} \mathrm{M}$ Ribo. ${ }^{1}$ Departament de Medicina, Universitat Autònoma de Barcelona; ${ }^{2}$ Stroke Unit, Neurology, Hospital Vall d'Hebron; ${ }^{3}$ Electronics and Biomedical Engineering, University of Barcelona; ${ }^{4}$ Biomaterials for Regenerative Therapies, Institute for Bioengineering in Catalonia; ${ }^{5}$ Neuroradiology, Hospital Vall d'Hebron, Barcelona; ${ }^{6}$ CIBER en Bioingeniería, Biomateriales y Nanomedicina, CIBER, Madrid; ' Materials Science and Engineering, Technical University of Catalonia, Barcelona, Spain

\subsection{6/neurintsurg-2021-ESMINT.49}

Introduction A direct aspiration first pass thrombectomy is a fast-growing technique for which a broad catalog of catheters that provide a wide range of aspiration forces can be used.

Objectives To characterize different catheters' aspiration performance on stiff clots in an in vitro vascular model.

Aims To demonstrate that labeled catheter inner diameter (labeled-ID) is not the only parameter that affects the aspiration force (asp-F) and that thrombus-catheter tip interaction and distensibility also play a major role.

Methods We designed an experimental setup consisting of a $3 \mathrm{D}$-printed carotid artery immersed in water. We measured asp-F and distensibility of catheter tips when performing ADAPT on a stiff clot analog larger than catheter labeled-ID. Correlations between asp-F, catheter ID, and tip distensibility were statistically assessed.

Results Experimental asp-F and catheter labeled-ID were correlated $(\mathrm{r}=0.9601 ; \mathrm{P}<0.01)$. The relative difference between 